\title{
Computed tomograpy evaluation of morphological changes, clinical response and survival in colorectal cancer liver metastasis treated by regorafenib: A case report
}

\author{
NOBUHISA MATSUHASHI, TAKAO TAKAHASHI, JUNKO KATO, TOSHIYUKI TANAHASHI, \\ SATOSHI MATSUI, YOSHIYUKI SASAKI, HISASHI IMAI, YOSHIHIRO TANAKA, NAOKI OKUMURA, \\ KAZUYA YAMAGUCHI, MANABU FUTAMURA, SHINJI OSADA and KAZUHIRO YOSHIDA
}

Department of Surgical Oncology, Gifu University School of Medicine, Gifu 501-1194, Japan

Received December 24, 2015; Accepted July 28, 2016

DOI: $10.3892 / \operatorname{mco} .2016 .1045$

\begin{abstract}
Pathological response to preoperative chemotherapy was recently found to be correlated with improved survival and has been proposed as a new outcome end-point following resection of liver metastases from colorectal cancer (CRC). It was demonstrated that, particularly after therapy with bevacizumab, CRC liver metastases decreased in size and underwent distinct morphological changes on computed tomography (CT). However, morphological changes in response to treatment with regorafenib have not yet been reported. A 74-year-old male patient with synchronous multiple liver and lung metastases from colon cancer was treated with regorafenib as a fifth-line therapy. CT imaging revealed a decrease in the tumor size and distinct morphological changes, namely homogeneous attenuation and sharp tumor-liver interface. The patient continued to take regorafenib for 8 months. Thus, regorafenib appeared to be effective as a last-line chemotherapy. In particular, the unique morphological changes of the metastases on CT imaging of may represent a method for evaluating the effects of CRC cancer therapy.
\end{abstract}

\section{Introduction}

Regorafenib (Stivarga, Bayer HealthCare Pharmaceuticals) is an orally administered multikinase inhibitor.Preclinical studies demonstrated that regorafenib targets cell signaling pathways involved in tumor formation and progression, including inhibition of protein kinases associated with angiogenesis [e.g., vascular endothelial growth factor receptor (VEGFR) 1-3 and TIE2], oncogenesis (e.g., KIT and RET), and maintainance

Correspondence to: Dr Kazuhiro Yoshida, Department of Surgical Oncology, Gifu University School of Medicine, 1-1 Yanagido, Gifu 501-1194, Japan

E-mail: kyoshida@gifu-u.ac.jp

Key words: morphological change, regorafenib, colorectal cancer liver metastases of the tumor microenvironment (e.g., platelet-derived growth factor receptor and fibroblast growth factor receptor) $(1,2)$.

At present, regorafenib has been approved for use following 3rd-line therapy for metastatic colorectal cancer (CRC) (3). As shown by computed tomography (CT) imaging, last-line chemotherapy is often ineffective. In the present case, treatment with regorafenib was indicated based on the presence of synchronous multiple lung and liver metastases from colon cancer and CT imaging revealed a significant change in tumor size. We herein report the patient's clinical course and CT imaging findings.

\section{Case presentation}

A 74-year-old male patient with sigmoid colon cancer and synchronous lung and liver metastases (stage IV) was treated with high anterior resection and D3 lymphadenectomy. The patient had undergone 16 courses of FOLFOX + bevacizumab (BV) as postoperative first-line therapy. Due to disease progression, follow-up treatment with 19 courses of BV + FOLFIRI was administered as a second-line therapy. The patient's performance status (PS) worsened to 2 due to the side effects of chemotherapy (general fatigue and edema). The disease continued to progress and the patient was administered 14 courses of panitumumab (an agent with anti-epidermal growth factor receptor activity in patients with wild-type Kras) as third-line chemotherapy, and the PS improved from 2 to 1. The disease progressed further and the patient underwent fourth-line chemotherapy comprising three courses of panitumumab plus irinotecan; however disease progression continued. Regorafenib was then administered as the last-line (fifth-line) therapy, the patient's side effects gradually subsided and the disease stabilized. The tumor size (liver and lung) decreased and morphological changes were revealed by $\mathrm{CT}$ imaging. In addition, the levels of the tumor markers carcinoembryonic antigen (CEA) and carbohydrate antigen (CA) 19-9 decreased in parallel with the changes in tumor morphology (Figs. 1-3). The patient continued to receive regorafenib for 8 months. As proteinuria $(+++)$ appeared during the clinical course, the dose of regorafenib was gradually reduced from 160 to $80 \mathrm{mg}$, while repeating stop-and-go therapy. Although the condition 


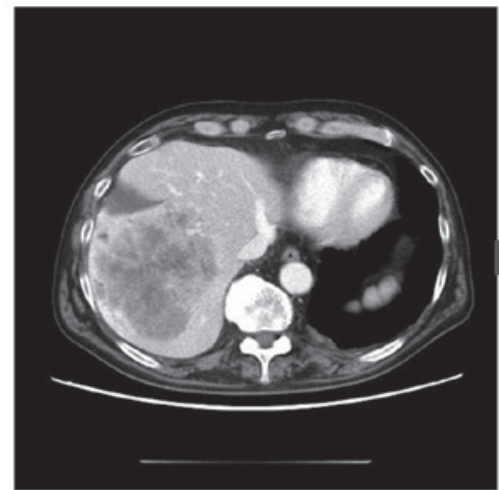

Prior to treatment

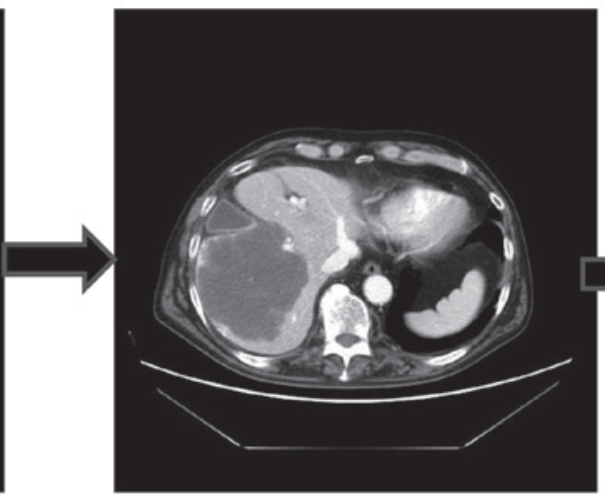

After 2 months

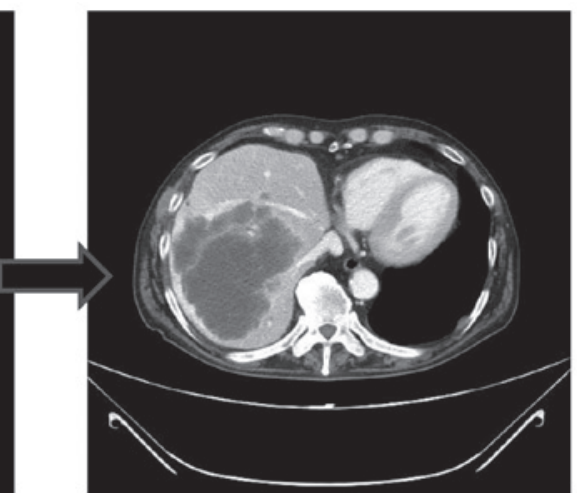

After 5 months
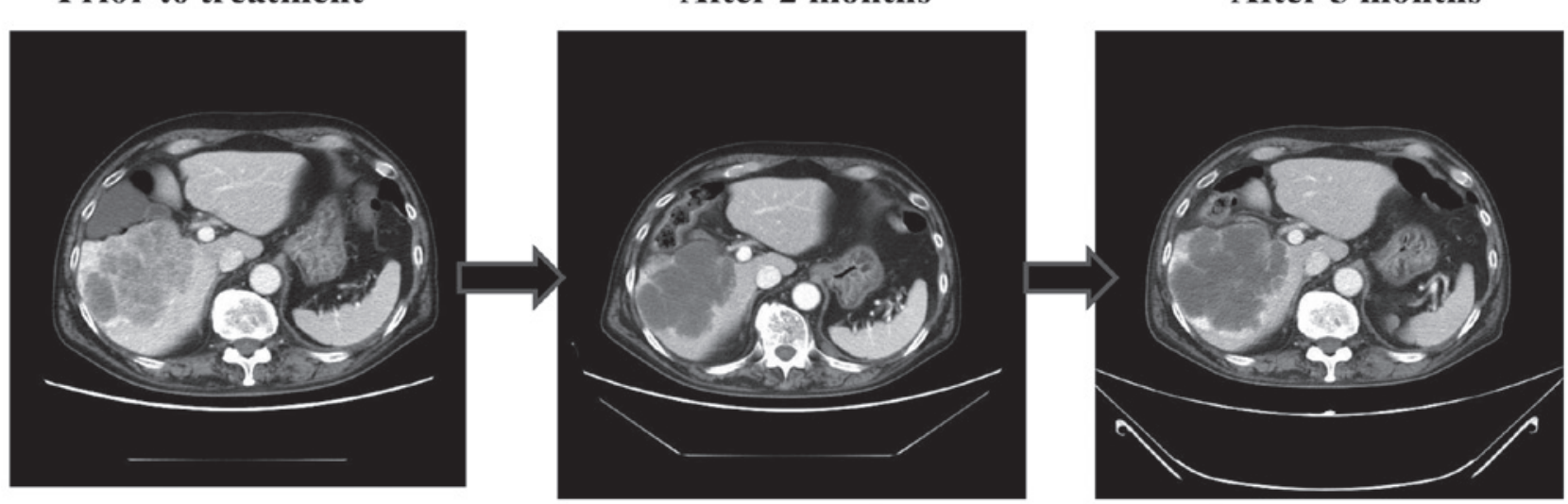

Figure 1. Clinical course: Computed tomography images showing morphological response of colorectal cancer liver metastasis to regorafenib treatment. The liver metastasis was $102 \mathrm{~mm}$ prior to regorafenib treatment, $100 \mathrm{~mm}$ after 2 months and $105 \mathrm{~mm}$ after 5 months of treatment. Top row, posterior area; bottom row, left lobe.

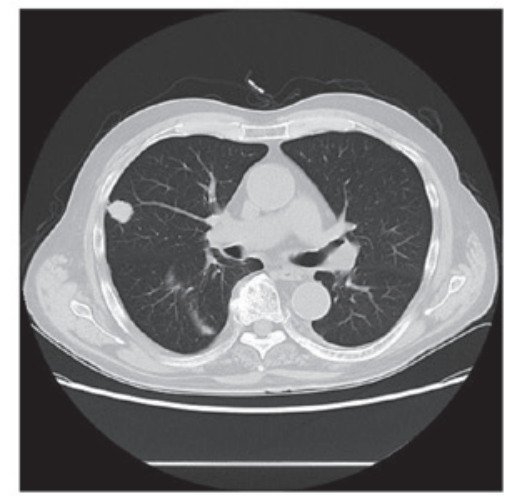

Prior to treatment

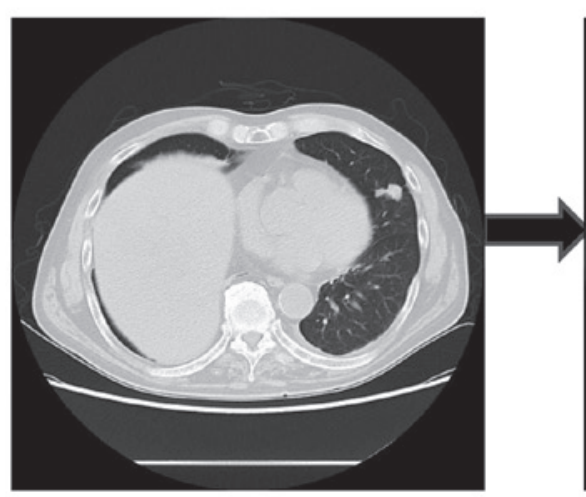

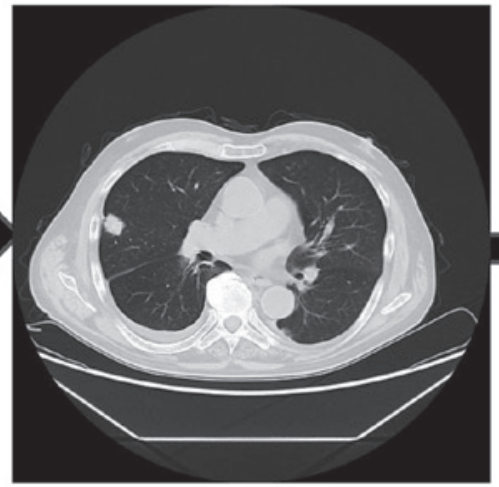

After 2 months

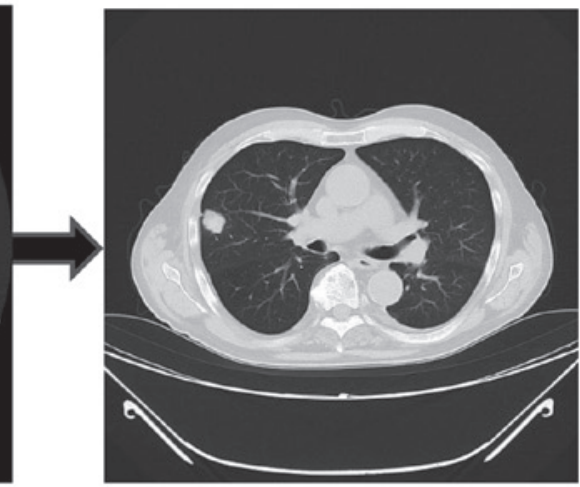

After 5 months

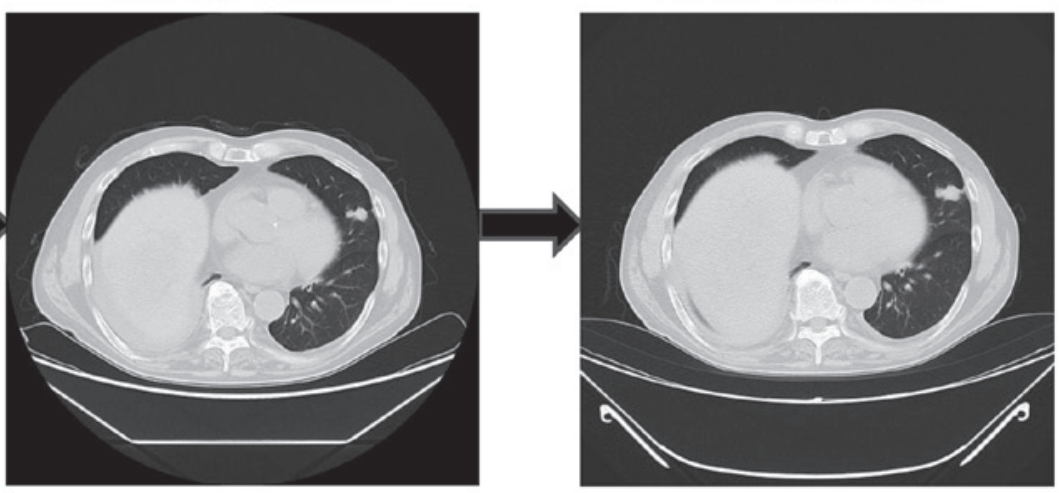

Figure 2. Clinical course: Computed tomography images of lung metastasis from colorectal cancer indicating stable disease. The right and left lung metastases were 17 and $16 \mathrm{~mm}$ prior to regorafenib treatment, 13 and $15 \mathrm{~mm}$ after 2 months, and 14 and $14 \mathrm{~mm}$ after 5 months of threatment, respectively. Top row, right lung metastases; bottom row, left lung metastases. 


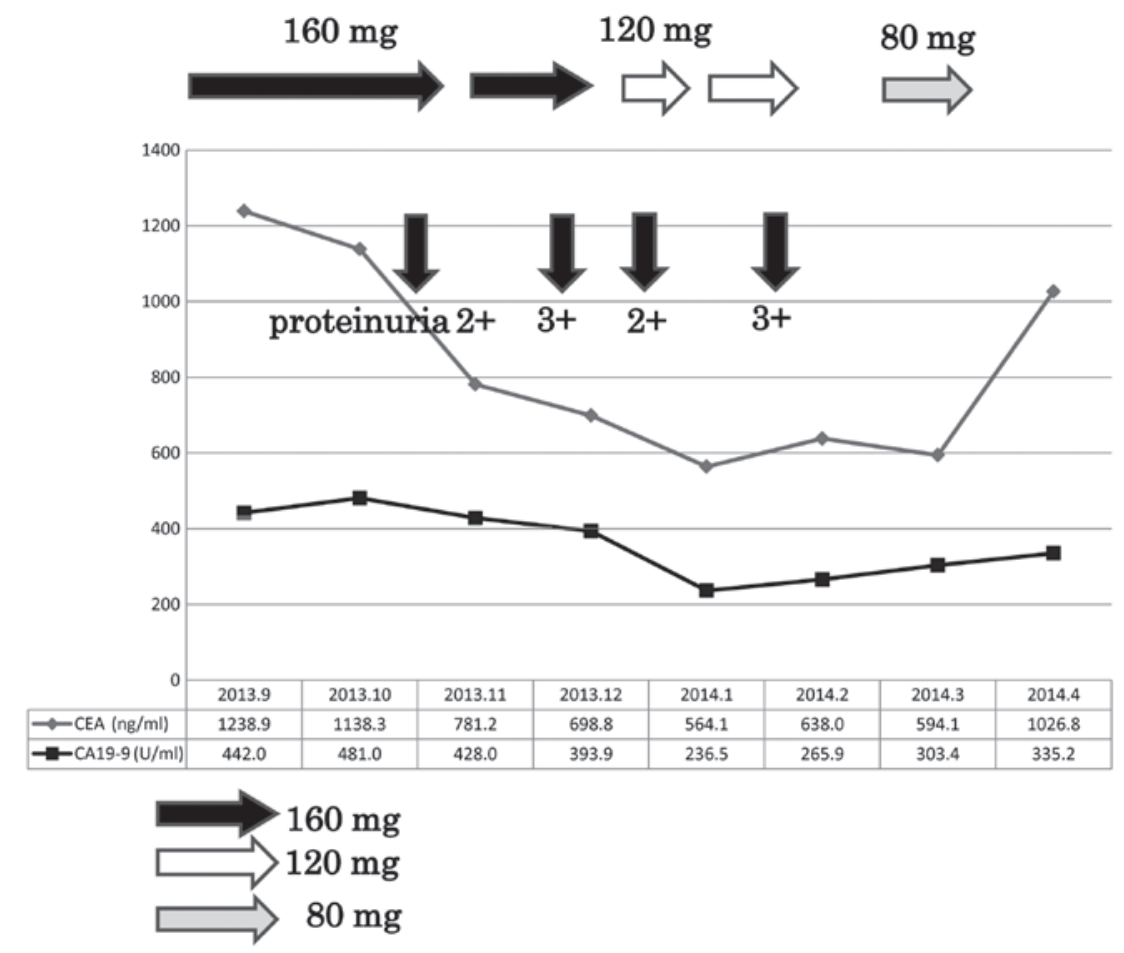

Figure 3. Tumor marker levels and proteinuria over the course of regorafenib treatment. CEA, carcinoembryonic antigen; CA19-9, carbohydrate antigen 19-9.

improved with the dose reduction and the stop-and-go therapy, nephrotic syndrome ultimately developed (Fig. 3). The patient eventually succumbed to nephrotic syndrome and cardiac failure from the original disease at 3 years and 10 months after the initiation of first-line chemotherapy.

Written informed consent was obtained from the patient for the publication of this case report and accompanying images.

\section{Discussion}

Chemotherapy has played a pivotal role in the multidisciplinary management of colorectal liver metastases (CLM). Systemic chemotherapy may reduce the size of metastases, increase their resectability, and may also help select patients who are most likely to benefit from surgery by assessing tumor response to chemotherapy $(4,5)$. However, the conventional tumor size-based radiological Response Evaluation Criteria in Solid Tumours (RECIST) may be inadequate for assessing the response to chemotherapy (6), particularly in patients treated with a regimen such as $\mathrm{BV}$, which interferes with angiogenesis. The pathological response to preoperative chemotherapy was recently found to be correlated with improved survival and has been proposed as a new outcome end-point following resection of CLM $(7,8)$. In particular, Shindoh et al (9) reported that, following therapy containing BV, the CLM tended to decrease in size and also underwent distinct morphological changes on CT, namely homogeneous attenuation and sharp tumor-liver interface. Recently, chemotherapy for CRC has markedly progressed. In particular, the treatment for advanced or metastatic CRC has significantly improved due to the development of the FOLFOX and FOLFIRI regimens. Furthermore, the introduction of targeted therapy has made the treatment of CRC patients more effective (10).
Regorafenib is the first small-molecule multikinase inhibitor to offer a survival benefit in metastatic CRC that has progressed after all standard therapies. In the CORRECT study, no patients achieved a complete response; however, 5 patients receiving regorafenib and 1 patient assigned to placebo exhibited a partial response, with objective response rates of 1.0 and $0.4 \%$, respectively $(\mathrm{P}=0.19)$ (3). As complete and partial responses were obtained in only a few patients, regorafenib is unlikely to achieve complete or partial disease response. However, disease control was achieved in $41 \%$ of the patients assigned regorafenib and $15 \%$ of the patients assigned a placebo $(\mathrm{P}<0.0001)$. The median duration of stable disease was 2.0 months in the regorafenib group and 1.7 months in the placebo group. These data indicate that regorafenib as last-line chemotherapy is unlikely to produce effective results on imaging (RECIST, version1.1) (6). However, the results of the present study indicated that evaluation of liver metastases by CT may be effective (10).

The end-point in cancer research is overall survival; however, tumor response and time to progression are considered pivotal for surrogate assessment of treatment efficacy. Tumor response was initially assessed according to the World Health Organization (WHO) criteria, and later according to the RECIST guidelines (11). WHO and RECIST define standard measurement methods for converting radiological observations into a quantitative and statistically tractable framework for measuring the response of tumor size to therapy. Both methods offer simple approaches to determining anatomical size and changes during treatment as indicators of response $(12,13)$. Target lesions may be measured with either the bilinear product approach (WHO) or the single linear summation (RECIST) (14-16). Of note, following anti-VEGF antigen-containing therapy, CRC metastases not only tend to decrease in size, but also undergo distinct morphological changes on CT (9). 
Initially, the morphological changes on CT in our patient indicated a response to regorafenib. These changes may prolong overall survival and improve the quality of life. However, in a number of patients, morphological changes do not result in any change in tumor size; therefore, they are not suitable for evaluation according to RECIST. Evaluation of chemotherapeutic response following regorafenib and anti-VEGF antigen therapy were included in the modified RECIST (mRECIST) guidelines, which were developed to assess response in patients with hepatocellular carcinoma based on the measurement of viable tumor with arterial enhancement on CT. The guidelines of mRECIST were evaluated and compared with those in RECIST for patients who received sorafenib for advanced hepatocellular carcinoma. The mRECIST guidelines for hepatocellular carcinoma introduced amendments to RECIST for the determination of tumor response in target lesions. The majority of the patients with stable disease according to RECIST had a different prognosis according to mRECIST. We recommend that a suitable CT scan evaluation according to mRECIST guidelines is performed in patients with CLM. mRECIST should be used for the standard assessment of treatment efficacy, particularly in patients receiving regorafenib.

In conclusion, regorafenib appeared to be effective as a last-line chemotherapy treatment in a patient with sigmoid colon cancer and synchronous lung and liver metastases. Identifying unique morphological changes of metastatic lesions on CT imaging may be a suitable method for evaluating the effects of treatment in CRC.

\section{References}

1. Strumberg D, Scheulen ME, Schultheis B, Richly H, Frost A, Büchert M, Christensen O, Jeffers M, Heinig R, Boix O and Mross K: Regorafenib (BAY 73-4506) in advanced colorectal cancer: A phase I study. Br J Cancer 106: 1722-1727, 2012.

2. Mross K, Frost A, Steinbild S, Hedbom S, Büchert M, Fasol U, Unger C, Krätzschmar J, Heinig R, Boix O and Christensen O: A phase I dose-escalation study of regorafenib (BAY 73-4506), an inhibitor of oncogenic, angiogenic, and stromal kinases, in patients with advanced solid tumors. Clin Cancer Res 18: 2658-2667, 2012.

3. Grothey A, Van Cutsem E, Sobrero A, Siena S, Falcone A, Ychou M, Humblet Y, Bouché O, Mineur L, Barone C, et al: Regorafenib monotherapy for previously treated metastatic colorectal cancer (CORRECT): An international, multicentre, randomised, placebo-controlled, phase 3 trial. Lancet 381: 303-312, 2013.

4. Sorbye H, Mauer M, Gruenberger T, Glimelius B, Poston GJ, Schlag PM, Rougier P, Bechstein WO, Primrose JN, Walpole ET, et al: Predictive factors for the benefit of perioperative FOLFOX for resectable liver metastasis in colorectal cancer patients (EORTC Intergroup Trial 40983). Ann Surg 255: 534-539, 2012.

5. Schwarz L, Michel P and Scotté M: Predictive factors for the benefit of perioperative FOLFOX for resectable liver metastasis in colorectal cancer patients (EORTC Intergroup Trial 40983). Ann Surg 261: e28-e29, 2015.
6. Eisenhauer EA, Therasse P, Bogaerts J, Schwartz LH, Sargent D, Ford R, Dancey J, Arbuck S, Gwyther S, Mooney M, et al: New Response Evaluation Criteria in Solid Tumours: Revised RECIST guideline (version 1.1). Eur J Cancer 45: 228-247, 2009.

7. Adam R, Wicherts DA, de Haas RJ, Aloia T, Lévi F, Paule B, Guettier C, Kunstlinger F, Delvart V, Azoulay D and Castaing D: Complete pathologic response after preoperative chemotherapy for colorectal liver metastases: Myth or reality? J Clin Oncol 26: 1635-1641, 2008.

8. Rubbia-Brandt L, Giostra E, Brezault C, Roth AD, Andres A, Audard V, Sartoretti P, Dousset B, Majno PE, Soubrane O, et al: Importance of histological tumor response assessment in predicting the outcome in patients with colorectal liver metastases treated with neo-adjuvant chemotherapy followed by liver surgery. Ann Oncol 18: 299-304, 2007.

9. Shindoh J, Loyer EM, Kopetz S, Boonsirikamchai P, Maru DM, Chun YS, Zimmitti G, Curley SA, Charnsangavej C, Aloia TA and Vauthey JN: Optimal morphologic response to preoperative chemotherapy: An alternate outcome end point before resection of hepatic colorectal metastases. J Clin Oncol 30: 4566-4572, 2012.

10. Chun YS, Vauthey JN, Boonsirikamchai P, Maru DM, Kopetz S, Palavecino M, Curley SA, Abdalla EK, Kaur H, Charnsangavej $\mathrm{C}$ and Loyer EM: Association of computed tomography morphologic criteria with pathologic response and survival in patients treated with bevacizumab for colorectal liver metastases. JAMA 302: 2338-2344, 2009.

11. Therasse P, Arbuck SG, Eisenhauer EA, Wanders J, Kaplan RS, Rubinstein L, Verweij J, Van Glabbeke M, van Oosterom AT, Christian MC and Gwyther SG: New guidelines to evaluate the response to treatment in solid tumors. European Organization for Research and Treatment of Cancer, National Cancer Institute of the United States, National Cancer Institute of Canada. J Natl Cancer Inst 92: 205-216, 2000

12. Matsuhashi N, Takahashi T, Tanahashi T, Matsui S, Sasaki Y, Tanaka Y, Okumura N, Yamaguchi K, Osada S and Yoshida K: The efficacy of 'Abound ${ }^{\mathrm{TM}}$ ', a nutritional supplement containing L-glutamine, L-arginine, citric acid, and calcium HMB, for skin disorders that developed as adverse drug reactions to anti-EGFR antibody preparation administration: Pilot study. Int J Colorectal Dis 31: 1055-1057, 2016.

13. Matsuhashi N, Takahashi T, Nonaka K, Ichikawa K, Yawata K,

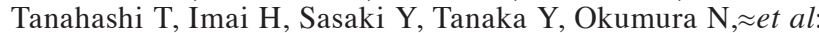
A case report on efficacy of Abound ${ }^{\mathrm{TM}}$ for anti-EGFR antibody-associated skin disorder in metastatic colon cancer. World J Surg Oncol 12: 35, 2014.

14. Ronot M, Bouattour M, Wassermann J, Bruno O, Dreyer C, Larroque B, Castera L, Vilgrain V, Belghiti J, Raymond E and Faivre S: Alternative Response Criteria (Choi, European Association for the Study of the Liver, and modified Response Evaluation Criteria in Solid Tumors [RECIST]) versus RECIST 1.1 in patients with advanced hepatocellular carcinoma treated with sorafenib. Oncologist 19: 394-402, 2014.

15. Edeline J, Boucher E, Rolland Y, Vauléon E, Pracht M, Perrin C, Le Roux C and Raoul JL: Comparison of tumor response by Response Evaluation Criteria in Solid Tumors (RECIST) and modified RECIST in patients treated with sorafenib for hepatocellular carcinoma. Cancer 118: 147-156, 2012.

16. Kawaoka T, Aikata H, Murakami E, Nakahara T, Naeshiro N, Tanaka M, Honda Y, Miyaki D, Nagaoki Y, Takaki S, et al: Evaluation of the mRECIST and $\alpha$-fetoprotein ratio for stratification of the prognosis of advanced-hepatocellular-carcinoma patients treated with sorafenib. Oncology 83: 192-200, 2012. 